\title{
Innervation zone distribution of the biceps brachii muscle examined using voluntary and electrically-evoked high-density surface EMG
}

\author{
Chengjun Huang ${ }^{1,2,3}$, Cliff S. Klein', Zhaojian Meng ${ }^{1}$, Yingchun Zhang ${ }^{4}$, Sheng Li ${ }^{2,3}$ and Ping Zhou ${ }^{2,3^{*}}$ (D)
}

\begin{abstract}
Background: High density surface electromyography (EMG) can be used to estimate muscle innervation zones (IZ). The objective of this study was to compare the differences in the distribution of the biceps brachii (BB) IZ derived from voluntary contractions (VC) and electrical stimulation (ES) of the musculocutaneous nerve.

Methods: Surface EMG signals were recorded from the medial and lateral BB with two 64-channel high density electrode matrices in eight healthy men. The surface EMG was recorded at different percentages of the maximal voluntary contraction (MVC) force (20-100\% MVC) and at different percentages of the current needed to elicit a maximal M-wave $\left(20-100 \% I_{\max }\right)$. The IZs of the medial and lateral BB were identified from the EMG signals and expressed as a row number within a given medial-lateral column.

Results: ES current intensity had no significant effect on the group mean IZ location $(p>0.05)$. However, The IZ during VC was located more proximally with increasing force $(p<0.05)$, likely due to muscle shortening. The position of the IZ varied slightly (by up to $\sim 8 \mathrm{~mm}$ ) in a medial-lateral direction under both contraction types, but this spatial effect was not significant. The IZ during ES and weak VC (20,40\% MVC) was similar $(p>0.05)$, but was more proximal in the latter than the former during 60-100\% MVC $(p<0.05)$.

Conclusion: ES can be used to detect spatial differences in IZ location free of the confounding effects of muscle shortening and recruitment order of different sized motor units. The method may prove beneficial for locating the IZ in patients who lack voluntary control of their musculature.
\end{abstract}

Keywords: EMG, Innervation zone, High-density electrode matrices, Electrical stimulation, Voluntary contraction

\section{Introduction}

Muscle spasticity often occurs in patients with neurological damage such as stroke and can have a negative impact on motor function [1]. It has been demonstrated that intramuscular injection of botulinum neurotoxins (BTX) is an effective and relatively safe treatment for spasticity $[2,3]$. However, there are side effects including muscle weakness, blocking of autonomic nerves, and muscle atrophy, which may relate to the toxin dosage

\footnotetext{
*Correspondence: ping.zhou.1@uth.tmc.edu

${ }^{2}$ Department of Physical Medicine and Rehabilitation, University of Texas Health Science Center at Houston, Houston, TX, USA

${ }^{3}$ TIRR Memorial Hermann Research Center, 1333B Moursund St, TIRR Research Building, Suite 326, Houston, TX 77030, USA

Full list of author information is available at the end of the article
}

[4]. The effectiveness of BTX treatment depends on the distance between the injection site and the location of the neuromuscular junctions $[5,6]$, which tend to cluster in a relatively narrow band termed the innervation zone (IZ) [7]. Therefore, it is of clinical importance to determine the IZ location, as this may help to optimize BTX dosage.

Motor unit action potentials (MUAPs) propagate in opposite directions from the neuromuscular junctions toward the muscle tendons. Surface electromyography (EMG) signals recorded by linear arrays or a matrix of electrodes have been widely used to identify the IZ location in many lower and upper limb muscles [7-10]. When surface EMG signals are recorded in single differential

(c) The Author(s). 2019 Open Access This article is distributed under the terms of the Creative Commons Attribution 4.0 International License (http://creativecommons.org/licenses/by/4.0/), which permits unrestricted use, distribution, and reproduction in any medium, provided you give appropriate credit to the original author(s) and the source, provide a link to the Creative Commons license, and indicate if changes were made. The Creative Commons Public Domain Dedication waiver (http://creativecommons.org/publicdomain/zero/1.0/) applies to the data made available in this article, unless otherwise stated. 
mode during voluntary contractions (VC), the IZ can be detected based either on a reversal in signal phase between two adjacent channels along the muscle fiber, or on the minimum amplitude in a single channel.

Usually, the IZ is determined by recording submaximal to maximal surface EMG during VC. However, this approach is not possible in patients who are unable to exert the necessary force because of paralysis or poor motor control. An alternative method for IZ location is to generate forces and associated EMG (i.e., M-wave) evoked by electrical stimulation (ES) [11].

One study used both VC and ES in the tibialis anterior of adults and found no significant differences in the IZ location between the two methods [11]. However, the surface EMG signals were detected using a single-column linear array, and thus could not detect the overall IZ distribution. Hence, it is uncertain whether $\mathrm{VC}$ and ES methods would also have detected the same IZ location in the medial and lateral portions of the muscle. Others have reported some variations in the IZ distribution in the mediallateral and proximal-distal directions of the biceps brachii (BB) based on VC $[12,13]$, but some conclude that IZ location is not altered across columns [10]. No one has addressed this issue comprehensively with ES. In the previous study of the tibialis anterior [11], IZ locations were obtained during low $\mathrm{VC}$ and $\mathrm{ES}$ force contractions only, equal to about $10 \%$ of the maximum voluntary contraction (MVC) force. Others have shown that the IZ location is dependent on the force level of the VC used to detect it [14, 15], but whether this is the case with different intensities of ES is unknown. It is possible that different groups of motor units (motor axons) with different EMG properties may be activated at low versus high stimulus intensities, with corresponding differences in the IZ location.

In this study, two matrices of electrodes were used to provide bi-dimensional spatial distribution of BB activity $[16,17]$. Surface EMG signals were collected under different VC levels and ES current intensities. The purpose of the study was to assess: 1) whether the BB IZ location shifts significantly with increases in ES current intensity; and 2) whether there are significant differences in IZ location between the VC and ES methods.

\section{Methods}

\section{Participants}

Eight healthy men between 20 and 33 years of age (mean $\pm \mathrm{SD}, 28.9 \pm 4.8$ years) volunteered to participate in the study. Their mean height and weight were $171.5 \pm 7.5 \mathrm{~cm}$ and $65 \pm 12 \mathrm{~kg}$, respectively. None had a history of injuries to the upper limb, nor any neurological or cardiovascular complications. They were informed of the possible risks and discomfort of the experiments, and signed an informed consent approved by the local ethics committee (ethical approval number: GWIRC-AF/SC-07/2016.20).

\section{Instrumentation}

Two electrode matrices were used to record VC and ES surface EMG (ELSCH064NM2, Bioelettronica, Torino, Italy, Fig. 1a-b). Each electrode matrix consists of 64 electrodes with an $8 \mathrm{~mm}$ inter electrode distance (IED) arranged in a grid of 5 columns by 13 rows (one column consisted of 12 electrodes and the other four of 13 electrodes). A piece of double adhesive foam ( $1 \mathrm{~mm}$ thick), that contained cavities for electrode paste, was placed between the skin and matrix. Each of the cavities was filled with conductive gel to ensure proper electrodeskin contact. The VC and ES surface EMG were recorded by a signal amplifier in monopolar acquisition mode (EMG-USB2, sampling frequency of $2048 \mathrm{~Hz}, 12-$ bit A/D converter, Bioelettronica, Torino, Italy). The surface EMG was amplified 1000x and 100x during the VC and ES protocols, respectively.

For ES responses, a constant-current stimulator (DS7A, Digitimer, Herthfordshire, UK) and bar electrode $(3 \mathrm{~cm}$ inter-electrode spacing) were used to evoke BB Mwaves. The force during elbow flexion was sensed by a load cell (CZL-3 T, Leitai, Bengbu, China) attached to a table (Fig. 1c), and recorded by the EMG-USB2 device.

\section{Procedure}

Each subject sat in a chair with their back fully against the backrest, with the hip, knee, and ankles joints flexed about $90^{\circ}$. The dominant arm (right side in all cases) was positioned in a custom-made force measuring device (Fig. 1c). The elbow angle was set at $120^{\circ}$ degrees $\left(180^{\circ}=\right.$ full extension $)$ and the forearm was supinated. The load cell and wrist were tightly connected with an inelastic strap to measure the vertical force at the wrist resulting from contraction of the elbow flexor (BB) muscles. The height of the chair and the arm support could be adjusted for each subject individually.

The skin of each subject was first shaved and cleaned with alcohol to reduce the skin-electrode impedance. The BB was detected based on palpation during a mild contraction. The two electrode matrices were joined together, and placed so the columns were parallel to the muscle fibers, with Matrix 1 over the lateral head and Matrix 2 over the medial head of the BB (Fig. 1b). The matrices were positioned below the stimulating bar electrode, meaning that surface EMG was recorded from the distal three-quarters of the BB. The matrices were firmly fixed with elastic bandages wrapped around the upper arm. A ground electrode was placed at the elbow. 

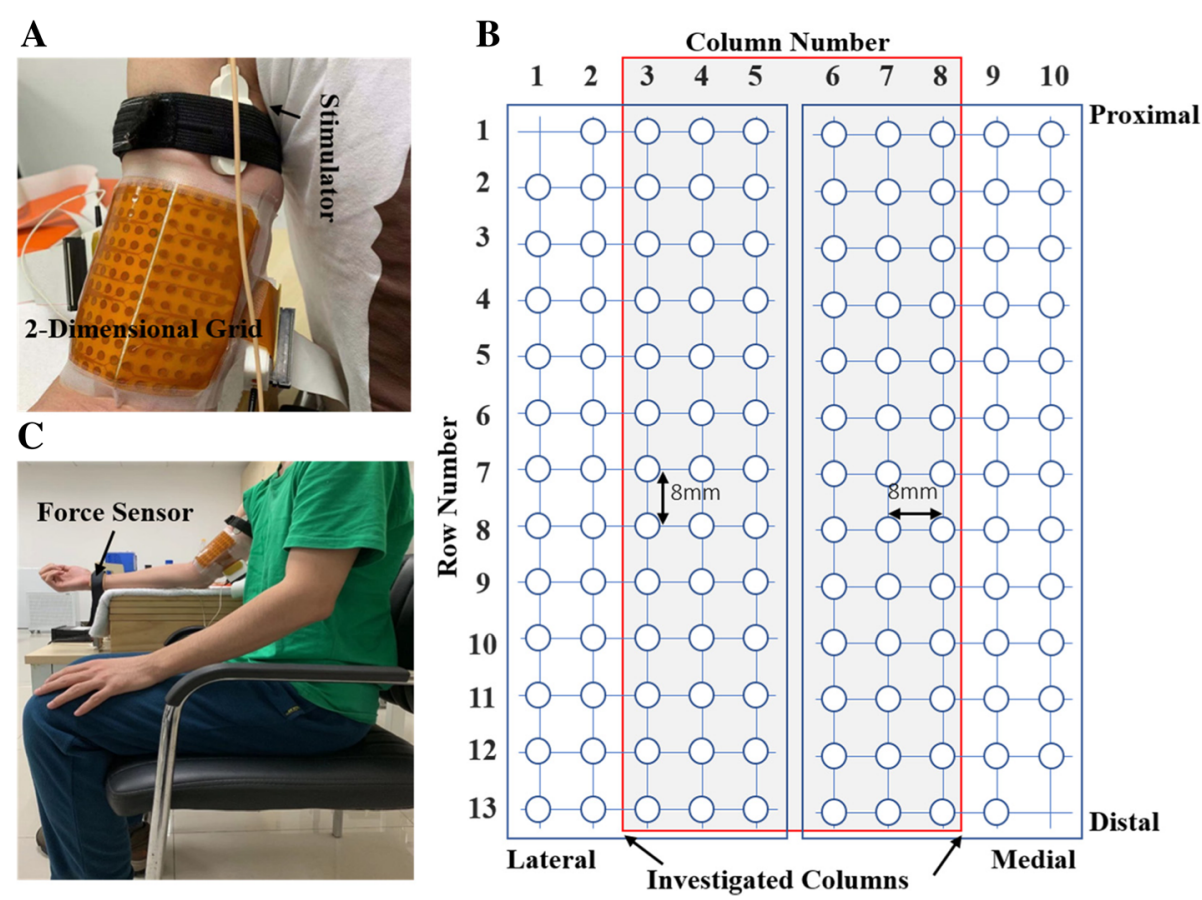

Fig. 1 a High-density electrode matrix consisting of a grid with 5 columns (that are positioned parallel to the muscle fiber direction) and 13 rows. b Schematic representation of the two adhesive 2D matrices. c Illustration of the experimental setup

\section{ES protocol}

The stimulation bar electrode was positioned vertically over the musculocutaneous nerve at the most proximal region of the BB. Single pulses (1 ms duration) were applied every $5 \mathrm{~s}$ at progressively greater current intensity (5-mA increments) until the M-wave peak-to-peak amplitude did not increase despite further increments in current [18]. The current intensity that evoked the maximum $\mathrm{M}$-wave $\left(\mathrm{M}_{\max }\right)$ was then recorded as the maximum current intensity $\left(\mathrm{I}_{\max }\right)$. Single pulses $(0.2 \mathrm{~Hz}, 1$ ms duration) at each of five different current intensities were then applied. The targeted current intensities were 20,4060 , and $80 \%$ and $100 \% \mathrm{I}_{\max }$ respectively.

\section{MVC and submaximal force protocol}

Each subject performed two to three MVCs and the largest of the trials was adopted as the MVC value. Subjects were strongly encouraged to give their best effort and were asked to maintain a stable shoulder, elbow, and wrist position. Each subject then completed a series of 5-s submaximal contractions at 20,40,60, and $80 \%$ MVC respectively. For each target, subjects performed the task 2 times with a 1-2-min rest between each to avoid muscle fatigue.

Force, as well as one selected M-wave channel from the EMG-USB2 system, were recorded to a second data collection system (1401 Plus, Cambridge Electronic Design, UK) and data collection software (Spike 2, Cambridge Electronic Design), and displayed on a second monitor in front of the subject. This was necessary for more immediate on-line feedback of target forces and M-waves compared to the processing speed of the EMG-USB2 system.

\section{Signal processing}

In some subjects, the surface EMG of the most two lateral and most two medial side columns were of low quality. The reasons might be that the electrodes of these columns were further way from the muscle compared to the more centrally located electrodes and/or poor electrode-skin contact. Therefore, these 4 columns were eliminated, leaving 6 columns for data analysis (Fig. 1b).

The monopolar signals of each column were postprocessed to single differential signals and then plotted (R2017a, The MathWorks Inc., MA, USA). As reported in previous studies $[7,14]$, the IZ was located either as the channel with the smallest signal amplitude or between the two adjacent channels whose signals were of opposite polarity. As the IED used in this study was $8 \mathrm{~mm}$, the spatial resolution for IZ determination was $4 \mathrm{~mm}$.

\section{Statistical analysis}

The distribution of data was tested using the KolmogorovSmirnov normality test. For both VC and ES conditions. A one-way repeated measure ANOVA was performed to determine the effect of $\mathrm{VC}$ level (\%MVC) or ES intensities $\left(\% \mathrm{I}_{\max }\right)$ on the IZ position, which was represented as the averaged row number (resolution 0.5) over all the columns. 
When a significant overall effect was confirmed, the Bonferroni-corrected Post-hoc test for multiple comparisons was done. The IZ row number obtained through $\mathrm{VC}$ and ES were compared using a Paired t-test. A $p$ value less than 0.05 was considered to be statistically significant. The analyses were performed using the SPSS software (SPSS, Chicago, IL).

\section{Results}

The IZ locations obtained through all VC and ES intensities showed normal distributions $(p>0.05)$. Typical surface EMG recordings of maximal $\mathrm{M}$-waves and $\mathrm{VC}$ at $60 \%$ MVC are shown for one subject (no. 8) in Fig. 2a-b, respectively. In these trials, the estimated IZs (arrows) were at row 5 or between rows 5 and 6 (i.e. row 5.5) for the six columns. The IZs in each column at the different ES and VC intensities of subject 8 are also shown (Fig. 3a-b). In this subject, the IZ for all ES and VC intensities ranged from row 4 to row 6 . The ES IZ within a particular column was unaffected by the stimulus intensity, but there were small IZ differences $(4 \mathrm{~mm})$ between columns (Fig. 3a). The VC IZ shifted proximally (up to $12 \mathrm{~mm}$ ) with increasing MVC percentage. There were only slight IZ differences between columns (up to $8 \mathrm{~mm}$ or 1 row, Fig. 3b). Given this, the IZ of the muscle was represented as the averaged row number over all the columns.

\section{ES-derived IZ}

Some subjects had no observable $\mathrm{M}$-wave at $20 \% \mathrm{I}_{\max }$. Therefore, only M-wave data from 40, 60, 80 and 100\% $I_{\max }$ are reported. There were small differences in the IZ at different stimulus intensities within a subject, but the differences were not consistent across subjects. Individual IZ at the different stimulus intensities, each averaged across the 6 columns, are shown in Fig. 4a. Overall, with the medium effect size, the IZ was found to be unrelated to stimulus intensity ( $p=0.672$, partial eta square $=0.07$, Table 1). The IZs for each column at $100 \% \mathrm{I}_{\max }$ are shown for each subject in Fig. 5a. There were small differences in the IZ between the different columns within a subject, but the differences were not consistent across all 8 subjects.

\section{VC-derived IZ}

Figure $4 \mathrm{~b}$ shows individual IZ at the different VC levels $(20,40,60,80$ and $100 \%$ MVC), each averaged across the 6 columns. In most instances, the IZ shifted proximally with increasing VC level. Overall, the IZ was significantly affected by the VC level $(p=0.012$, partial eta square $=$ 0.36 , Table 1). Post-hoc analysis indicated that the IZ was more proximal during the 60 and $80 \%$ MVC compared to the $20 \%$ MVC. The IZ was also more proximal during the $100 \%$ MVC compared to the $20 \%$ MVC, but the difference was not statistically significant. There were also differences in VC IZ between different columns within a subject, but the differences were not consistently observed across all subjects. The IZs for each column at 100\% MVC are displayed for each subject in Fig. 5b.

\section{VC vs. ES IZ location}

The mean $(N=8)$ IZs of all columns during VC and ES at the different $\mathrm{VC}$ levels and ES intensities are shown in Fig. 6 . The IZ during the $20 \%$ MVC was similar to the
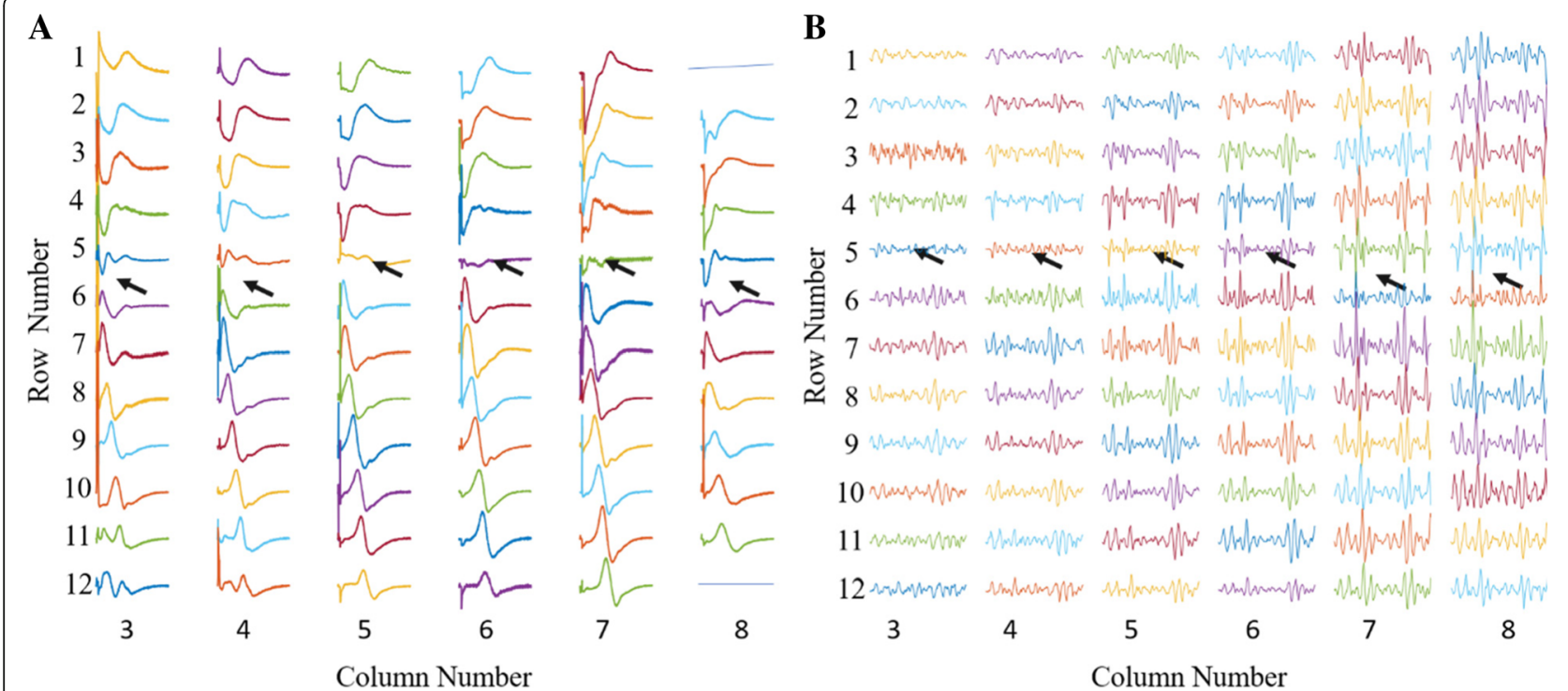

Fig. 2 Examples of IZ locations of the BB muscle from one subject (no. 8), based on ES evoked maximal M-waves (a) and a VC at 60\% MVC (b). Column 3 is the most lateral column and Row 1 is the most proximal row 

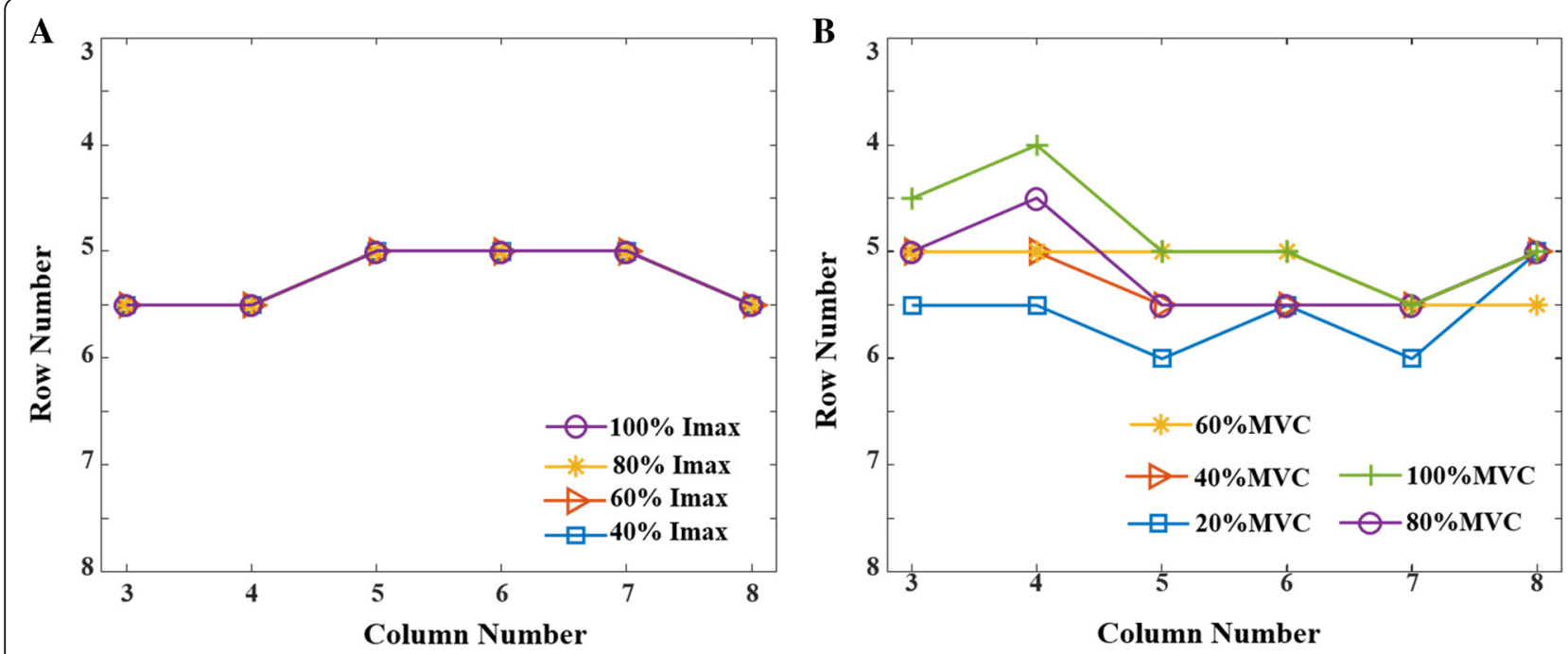

Fig. 3 IZ location (row number) of one representative subject (no. 8) in individual columns with increasing ES (a) or VC level (b)

ES IZ ( $p>0.05$, Table 2, paired t-test). However, the IZs for the higher VC levels were located more proximally compared to ES IZs, and the differences were significant for the three highest VC levels (60, 80, and 100\% MVC).

\section{Discussion}

The aim of the present study was to estimate and compare BB IZ distributions under different ES current intensities and VC levels with two electrode matrices. The BB was chosen as it plays an important role in upper limb function. It is often affected by spasticity in patients with neurological disorders, and thus is a useful model for assessing efficacy of various treatments [19].

\section{Detection of the IZ during ES}

In the ES protocol, we examined whether the detected IZ differed between low and high stimulus intensities and between columns (see section B below). We found the IZ on average was unrelated to stimulus intensity (Fig. 4a and Table 1).

There are a number of possible reasons why the IZ was unrelated to stimulus intensity. During ES, the Mwave is largely completed before the muscle shortens. Hence, any differences in muscle shortening due to differences in stimulus intensity likely had little impact on the recorded IZ. Previously, the IZ was found to shift proximally with increasing VC levels [13, 19], and this was confirmed in the present study. This shift during
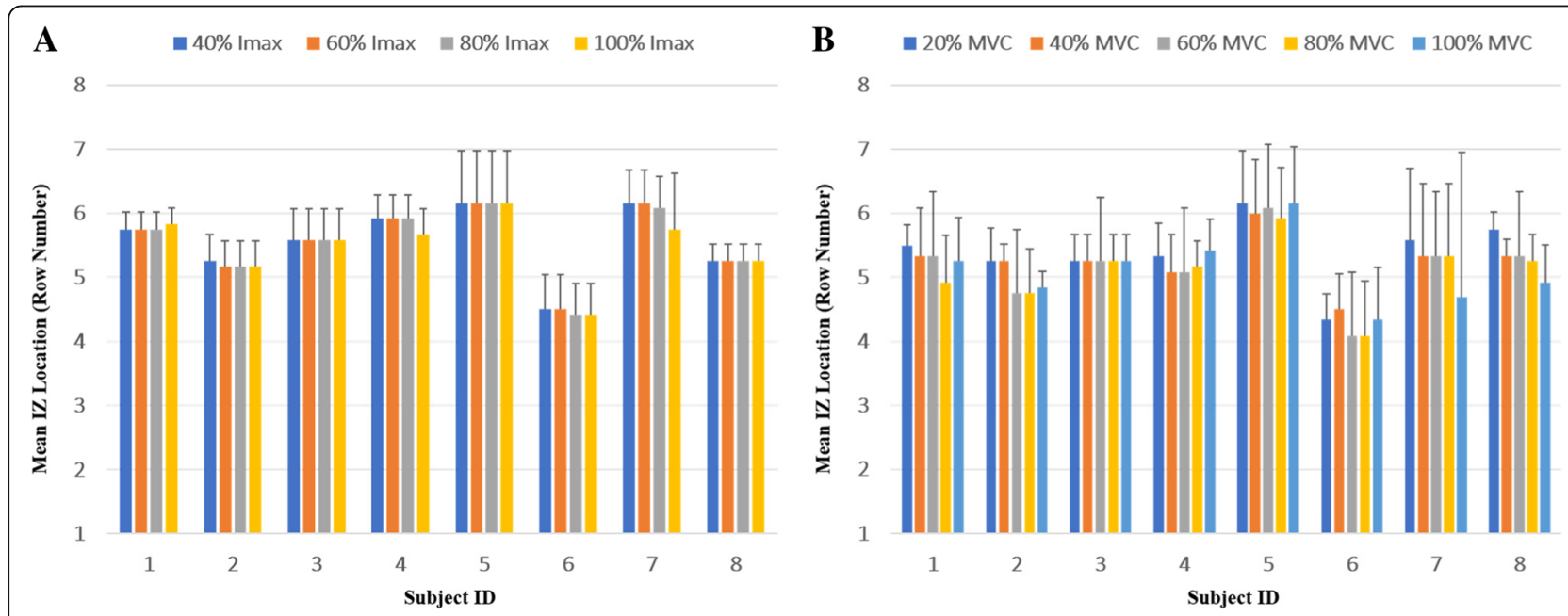

Fig. 4 Mean (SD) IZ location (row number) of all six columns at different ES intensities (a) and VC levels (b) for each of the 8 subjects 
Table 1 ANOVA on IZ position with VC level $(20,40,60,80$ and $100 \%$ MVC) or ES current intensities (40,60, 80 and 100\% Imax) as fixed factors

\begin{tabular}{lllll}
\hline Method & Factors & $\mathrm{F}$ & $\mathrm{P}$ & Effect Size (partial Eta square) \\
\hline ES & $\% I_{\max }$ & 0.522 & 0.672 & 0.07 \\
VC & $\% \mathrm{MVC}$ & 3.934 & 0.012 & 0.36 \\
\hline
\end{tabular}

VC likely reflects muscle shortening relative to the recording electrodes.

Another explanation for the lack of stimulus intensity on the IZ may relate to the recruitment order and location of different sized motor axons (motor units). During electrical nerve stimulation, progressive increases in stimulus intensity activate axons according to their size, with larger axons activated before smaller axons [20,21], although some also found that smaller axons were activated first, similar to voluntary contractions [22-24]. In addition, for both the vastus lateralis and biceps brachii, it has been found that smaller motor units tend to be located deeper in the muscle, whereas larger motor units tend to be located more superficially $[25,26]$. The IZ detected by high-density surface electrodes reflects the activity of more superficial muscle fibers [27]. With increasing ES intensity, the added recruitment of the smaller deeper MUs may have relatively little effect on the IZ location. In contrast to orderly recruitment according to axon size, some have argued that electrical nerve stimulation in-vivo activates axons randomly [28-30]. With random recruitment, the location of motor units is secondary and there may be a consistent proportional contribution of small and large motor units to the IZ, regardless of ES intensity. Our findings suggest that the IZ is independent of stimulus intensity, possibly because IZ detection was not confounded by muscle shortening and recruitment order of different sized motor units.
IZ distribution across different columns and subjects Both the results of the ES and VC protocols indicated that the IZ on average was unrelated to column location. This is consistent with the previous study, which found that IZ location did not change across columns [10]. However, there were some between-column differences in the IZ within individual subjects. For example, in subject 5 , the IZ of column 7 is more proximal than the other columns during both VC and ES (Fig. 5). In contrast, in subject 3, the IZ of column 8 is more distal than the other columns. These individual column-related differences in IZ may reflect normal biological variability of IZ location within the medial and lateral head of the BB. Other studies also found the IZ could differ between subjects. For example, Saitou et al. [7] demonstrated that the distribution of motor unit IZs varied substantially between subjects in both upper and lower limb muscles under VC. Another study also observed that the location of the main IZ was highly variable along the $\mathrm{BB}$ muscle belly between subjects [14]. Botter et al. [31] investigated the uniformity of IZ location for lower limb muscles in healthy subjects using ES and showed IZ inter-individual differences.

To conclude, we have shown that a two-dimensional electrode matrix can characterize spatial differences of IZ in the medial-lateral direction of the muscle [12], unlike a single electrode array. The demonstrated intersubject variability of the IZ is of great importance for individual determination of IZ locations prior to clinical treatments such as BTX injection.

\section{Concordance of IZ location between VC and ES}

The average IZ during the 20 and $40 \%$ MVC was similar to the IZ derived by ES (Table 2). This is consistent with a previous study that found a similar IZ during a $10 \%$ MVC and low intensity ES of the tibialis anterior [10]. However, we found that the IZ tended to be more
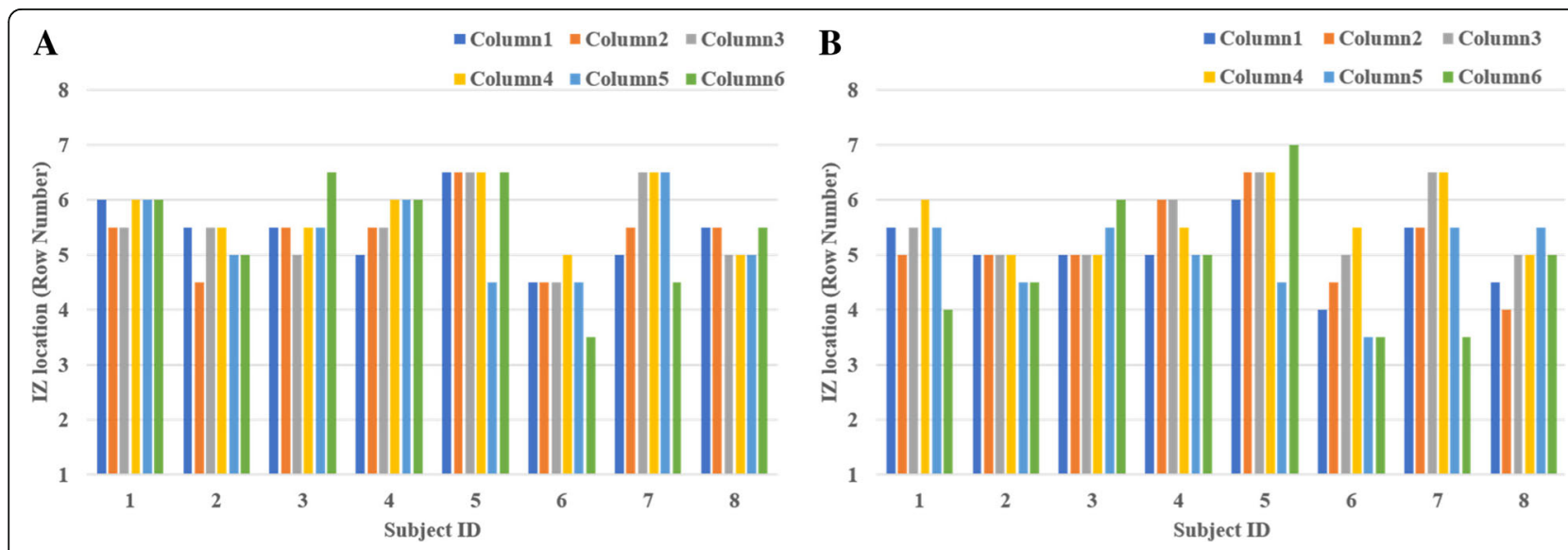

Fig. 5 The IZ for each column at 100\% I max (a) and 100\% MVC (b) for each subject 


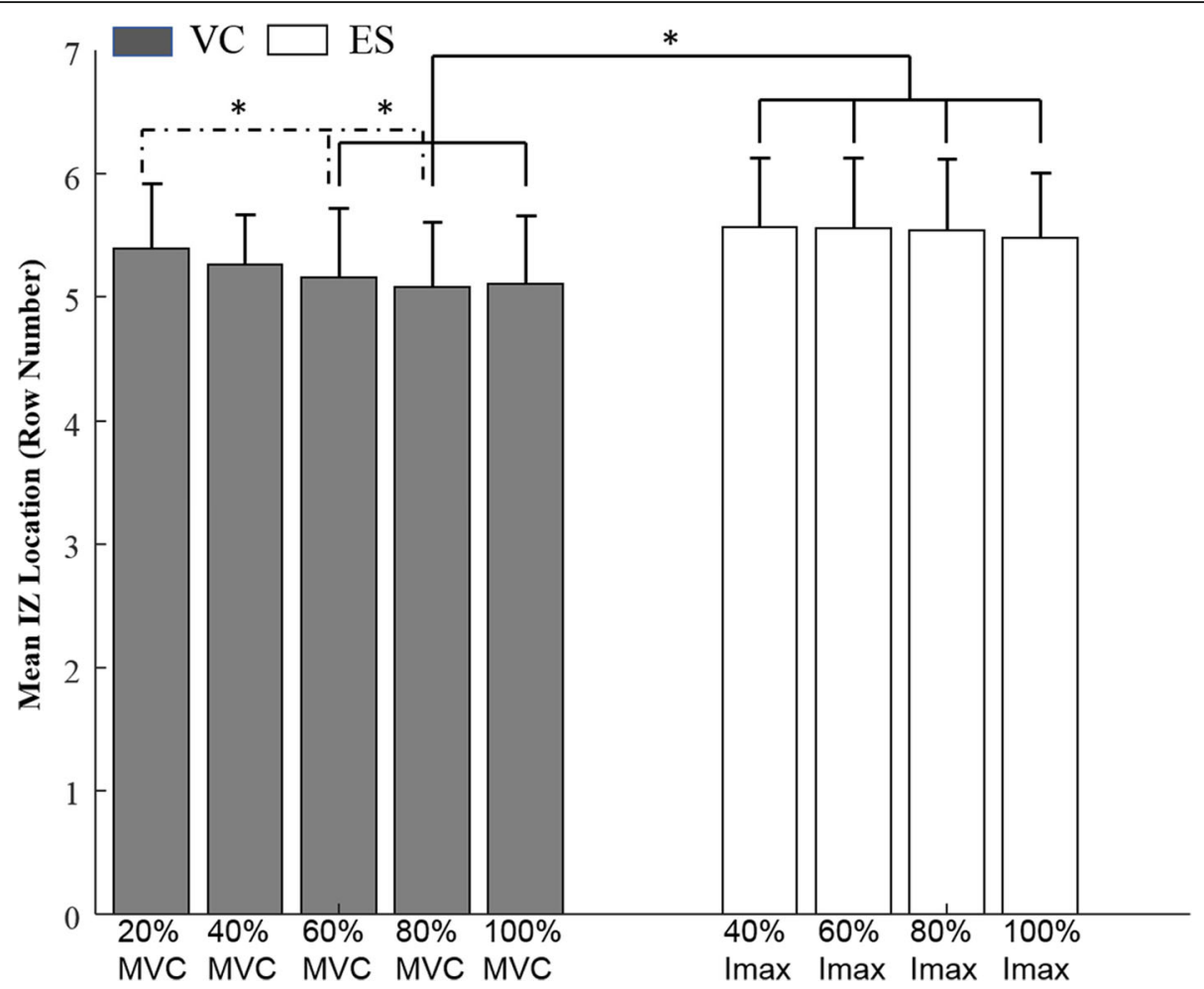

Fig. 6 Group mean (SD) IZ of all six columns at different VC levels (grey) and different ES intensities (white). * Significant difference between ES and $\mathrm{VC}$ derived $\mathrm{IZ}$ as well as between different $\mathrm{VC}$ intensities $(P<0.05)$

proximal during strong VC (60-100\% MVC, Table 2) compared to ES (Figs. 4b and 6).

One explanation for the more proximal IZ location during strong VC is likely muscle shortening as mentioned above, and discussed by others [13]. During voluntary contractions, the IZ is observed while the muscle is contracted. On the contrary, during a single pulse stimulation, the IZ is observed before the muscle begins to twitch. Therefore, a more distal IZ should be expected in electrically elicited contractions. Another possible explanation for the more proximal IZ during VC compared to ES may relate to the opposite order of motor unit recruitment under the two types of contractions [24].

Table 2 Paired t- test $p$-values and Cohen's d values for comparison of VC and ES IZ row number at different contraction levels and stimulus intensities: $p$-value (Cohen's d value)

\begin{tabular}{lllll}
\hline Contraction Levels & $40 \% \operatorname{Imax}$ & $60 \% \operatorname{Imax}$ & $80 \% \operatorname{Imax}$ & $100 \% \operatorname{Imax}$ \\
\hline $20 \%$ MVC & $0.351(0.35)$ & $0.267(0.43)$ & $0.270(0.42)$ & $0.270(0.42)$ \\
$40 \%$ MVC & $0.067(0.76)$ & $0.060(0.79)$ & $0.065(0.81)$ & $0.056(0.81)$ \\
$60 \%$ MVC & $\mathbf{0 . 0 1 2}(1.18)$ & $\mathbf{0 . 0 0 7}(1.32)$ & $\mathbf{0 . 0 0 6}(1.37)$ & $\mathbf{0 . 0 0 7}(1.37)$ \\
$80 \%$ MVC & $\mathbf{0 . 0 0 7}(1.32)$ & $\mathbf{0 . 0 0 5}(1.45)$ & $\mathbf{0 . 0 0 4}(1.48)$ & $\mathbf{0 . 0 0 4}(1.48)$ \\
$100 \%$ MVC & $\mathbf{0 . 0 1 9}(1.08)$ & $\mathbf{0 . 0 1 9}(1.09)$ & $\mathbf{0 . 0 1 5}(1.13)$ & $\mathbf{0 . 0 1 5}(1.13)$ \\
\hline
\end{tabular}

Entries in boldface indicate a statistically significant difference $(p<0.05)$
Hence, relatively larger superficially located motor units would be activated as VC force increased compared to ES.

\section{Clinical relevance}

ES has advantages for estimating IZ, particularly in light of our finding that it is independent of stimulus intensity. Detecting the IZ by sub-maximal ES may be preferred for certain situations including patients with paralysis and for BTX injection. ES also revealed subtle medial-lateral differences in IZ between subjects that can be located with a matrix. This may be of practical value for optimal BTX dosage, at least for superficial fibers.

\section{Limitations}

There are a few limitations in the present study that should be considered for further work in this area. Firstly, the IED of the two matrices used in this study was $8 \mathrm{~mm}$. This distance corresponds to a spatial resolution for IZ detection of $4 \mathrm{~mm}$, relatively large compared to others $[11,15]$. Hence, our method may not detect potentially smaller (i.e., $2-3 \mathrm{~mm}$ ) medial-lateral or intensity-related differences in IZ. Automatic detection algorithms and signal processing [32] could be used to locate the IZ and might improve the resolution. Secondly, the number of subjects studied was only 8 , which is relatively small compared with previous studies. The 
partial Eta square showed that for ES, the effect size is only medium. More subjects will be helpful to precisely investigate the effect of different ES current levels on the IZ location. Thirdly, each subject was studied only once in this study. Further tests are necessary to determine repeatability of the recordings. In addition, we only investigated IZ location in healthy subjects, and it would be interesting to investigate how the findings may differ in patients with neurological disorders.

\section{Conclusions}

We investigated two methods (VC and ES) for detecting the BB IZ in healthy subjects. The results demonstrated that: 1) there might be no effect of ES intensity on IZ location; 2) there were some spatial differences in IZ within individual subjects; 3) IZ locations were similar between ES and low-level VC. Detection of the IZ using ES and electrode arrays is non-invasive, and convenient. Complementary studies are needed to assess the IZ in patient populations. Testing the two methods of IZ detection on patients with spasticity may be useful to optimize BTX dosage.

\section{Acknowledgements}

Not applicable.

\section{Authors' contributions}

Study concept and design: PZ, CK. YC, SL; Data collection: CH, CK, ZM; Data analysis and interpretation: $\mathrm{CH}, \mathrm{CK}, \mathrm{YZ}, \mathrm{SL}, \mathrm{PZ}$; Manuscript writing: $\mathrm{CH}, \mathrm{CK}, \mathrm{PZ}$; Study supervision: PZ, CK. All authors read, revised and approved the final manuscript.

\section{Funding}

This work was supported in part by the Research Funds of the Guangdong Work Injury Rehabilitation Center/Hospital (CH, CK, and ZM), and in part by the National Institutes of Health of the U.S. Department of Health and Human Services under Grants R01NS080839 and R01HD092389 (PZ). The funders had no role in study design, data collection and analysis, decision to publish, or preparation of the manuscript.

\section{Availability of data and materials}

The authors confirm that all data underlying the findings are fully available without restriction. Data are available from the Guangdong Work Injury Rehabilitation Center Institutional Data Access/Ethics Committee for researchers who meet the criteria for access to human subject data.

\section{Ethics approval and consent to participate}

The study was approved by the research ethics committee of Guangdong Work Injury Rehabilitation Center. All participants gave written informed consent in accordance with the Declaration of Helsinki.

\section{Consent for publication}

Not applicable.

\section{Competing interests}

The authors declare that they have no competing interests.

\section{Author details}

'Guangdong Work Injury Rehabilitation Center, Guangzhou, Guangdong, China. ${ }^{2}$ Department of Physical Medicine and Rehabilitation, University of Texas Health Science Center at Houston, Houston, TX, USA. ${ }^{3}$ TIRR Memorial Hermann Research Center, 1333B Moursund St, TIRR Research Building, Suite 326, Houston, TX 77030, USA. ${ }^{4}$ Department of Biomedical Engineering, University of Houston, Houston, TX, USA.
Received: 11 December 2018 Accepted: 28 May 2019

Published online: 11 June 2019

\section{References}

1. Chang SH, et al. Spasticity, weakness, force variability and sustained spontaneous motor unit discharges of resting spastic-paretic biceps brachii muscles in chronic stroke. Muscle Nerve. 2013:48(1):85-92.

2. Setler PE. Therapeutic use of botulinum toxins: background and history. Clin J Pain. 2002;18(6 Suppl):S119.

3. Simpson DM, et al. Assessment: botulinum neurotoxin for the treatment of movement disorders (an evidence-based review): report of the therapeutics and technology assessment Subcommittee of the American Academy of neurology. Neurology. 2008:70(19):1699-706

4. Jahn R. Neuroscience. A neuronal receptor for botulinum toxin. Science. 2006;312(5773):540-1.

5. Shaari CM, Ira Sanders MD. Quantifying how location and dose of botulinum toxin injections affect muscle paralysis. Muscle Nerve. 1993; 16(9):964.

6. Lapatki BG, et al. Botulinum toxin has an increased effect when targeted toward the muscle's endplate zone: a high-density surface EMG guided study. Clin Neurophysiol. 2011;122(8):1611-6.

7. Saitou K, et al. Innervation zones of the upper and lower limb muscles estimated by using multichannel surface EMG. J Hum Ergol. 2000;29(1-2):35.

8. Masuda T, Miyano H, Sadoyama T. The position of innervation zones in the biceps brachii investigated by surface electromyography. IEEE Trans Biomed Eng. 1985;BME-32(1):36-42

9. Piccoli MB, et al. Innervation zone locations in 43 superficial muscles: toward a standardization of electrode positioning. Muscle Nerve. 2014;49(3): 413-21.

10. Barbero M, Merletti R, Rainoldi A. Atlas of muscle innervation zones: understanding surface electromyography and its applications: Springer Science \& Business Media; 2012; Springer-Verlag Mailand, Milan, Italy.

11. Guzmán-Venegas RA, et al. Concordance of the location of the innervation zone of the tibialis anterior muscle using voluntary and imposed contractions by electrostimulation. J Electromyography Kinesiol. 2016;27:18-23.

12. Masuda T, Sadoyama T. Topographical map of innervation zones within single motor units measured with a grid surface electrode. IEEE Trans Biomed Eng. 1988;35(8):623-8.

13. Masuda T, Miyano H, Sadoyama T. The distribution of myoneural junctions in the biceps brachii investigated by surface electromyography. Electroencephalogr Clin Neurophysiol. 1983;56(6):597-603.

14. Piitulainen $\mathrm{H}$, et al. Innervation zone shift at different levels of isometric contraction in the biceps brachii muscle. J Electromyography Kinesiol. 2009; 19(4):667-75.

15. Defreitas JM, et al. An examination of innervation zone movement with increases in isometric torque production. Clin Neurophysiol. 2008;119(12): 2795-9.

16. Rojas-Martínez M, Mañanas MA, Alonso JF, et al. J Neuroeng Rehabil. 2012; 9(85.10):1186

17. Drost $\mathrm{G}$, et al. Clinical applications of high-density surface EMG: a systematic review. J Electromyography Kinesiol. 2006;16(6):586-602.

18. Klein CS, et al. Voluntary activation failure contributes more to plantar flexor weakness than antagonist coactivation and muscle atrophy in chronic stroke survivors. J Appl Physiol. 2010;109(5):1337.

19. Guzmán-Venegas RA, Araneda OF, Silvestre RA. Differences between motor point and innervation zone locations in the biceps brachii. An exploratory consideration for the treatment of spasticity with botulinum toxin. J Electromyography Kinesiol. 2014;24(6):923-7.

20. Solomonow M. External control of the neuromuscular system. IEEE Trans Biomed Eng. 1984;BME-31(12):752-63.

21. Blair E, Erlanger J. A comparison of the characteristics of axons through their individual electrical responses. Am J Physiol Legacy Content. 1933; 106(3):524-64

22. Feiereisen $\mathrm{P}$, Duchateau J, Hainaut K. Motor unit recruitment order during voluntary and electrically induced contractions in the tibialis anterior. Exp Brain Res. 1997:114(1):117-23.

23. Vanderthommen M, Duchateau J. Electrical stimulation as a modality to improve performance of the neuromuscular system. Exerc Sport Sci Rev. 2007;35(4):180-5. 
24. Maffiuletti NA. Physiological and methodological considerations for the use of neuromuscular electrical stimulation. Eur J Appl Physiol. 2010; 110(2):223-34.

25. Knight C, Kamen G. Superficial motor units are larger than deeper motor units in human vastus lateralis muscle. Muscle Nerve. 2005;31(4):475-80.

26. Stålberg E. Macro EMG, a new recording technique. J Neurol Neurosurg Psychiatry. 1980;43(6):475-82.

27. Merletti R, Holobar A, Farina D. Analysis of motor units with high-density surface electromyography. J Electromyogr Kinesiol. 2008;18(6):879-90.

28. Bickel CS, Gregory CM, Dean JC. Motor unit recruitment during neuromuscular electrical stimulation: a critical appraisal. Eur J Appl Physiol. 2011;111(10):2399.

29. Knaflitz M, Merletti R, De Luca CJ. Inference of motor unit recruitment order in voluntary and electrically elicited contractions. J Appl Physiol. 1990;68(4): 1657-67.

30. Hamilton LD, et al. Electrical nerve stimulation modulates motor unit activity in contralateral biceps brachii during steady isometric contractions. J Neurophysiol. 2018;120(5):2603-13

31. Botter A, et al. Atlas of the muscle motor points for the lower limb: implications for electrical stimulation procedures and electrode positioning. Eur J Appl Physiol. 2011;111(10):2461.

32. Ullah $\mathrm{K}$, et al. Automatic detection of motor unit innervation zones of the external anal sphincter by multichannel surface EMG. J Electromyogr Kinesiol. 2014;24(6):860-7.

\section{Publisher's Note}

Springer Nature remains neutral with regard to jurisdictional claims in published maps and institutional affiliations.

Ready to submit your research? Choose BMC and benefit from:

- fast, convenient online submission

- thorough peer review by experienced researchers in your field

- rapid publication on acceptance

- support for research data, including large and complex data types

- gold Open Access which fosters wider collaboration and increased citations

- maximum visibility for your research: over $100 \mathrm{M}$ website views per year

At $\mathrm{BMC}$, research is always in progress.

Learn more biomedcentral.com/submissions 